\title{
GROUPS IN WHICH ALL SUBGROUPS OF INFINITE RANK ARE SUBNORMAL
}

\author{
LEONID A. KURDACHENKO \\ Algebra Department, Dnepropetrovsk University, Vul. Naukova 13, Dnepropetrovsk 50, Ukraine 49000 \\ and HOWARD SMITH \\ Department of Mathematics, Bucknell University, Lewisburg, PA 17837, U.S.A. \\ e-mail: howsmith@bucknell.edu
}

(Received 23 September, 2002; accepted 15 May, 2003)

\begin{abstract}
Let $G$ be a locally soluble-by-finite group in which every nonsubnormal subgroup has finite rank. It is proved that either $G$ has finite rank or $G$ is soluble and locally nilpotent (and even a Baer group). On the other hand, a group $G$ is constructed that has infinite rank and satisfies the given hypothesis, but does not have every subgroup subnormal.
\end{abstract}

2000 Mathematics Subject Classification. 20E15, 20F19.

1. Introduction. The following result is established in [5].

THEOREM 1 [5, Theorem 1.22]. Let $G$ be a group whose non-subnormal subgroups have finite rank. If $G$ is soluble then either $G$ is a Baer group or $G$ has finite rank.

We recall here that a Baer group is one in which every cyclic (and hence every finitely generated) subgroup is subnormal, and that a group $G$ has finite rank $r$, say, if every finitely generated subgroup of $G$ is $r$-generated. (Thus $G$ has finite special rank $r$.) In [3] the authors show that (in particular) a locally soluble-by-finite group $G$ in which every subgroup of infinite rank is subnormal of defect at most $d$, where $d$ is some fixed positive integer, is either nilpotent of bounded class or of finite rank. This result generalizes to some extent the well-known theorem of Roseblade [9] that a group in which every subgroup is subnormal of bounded defect is nilpotent (of bounded class). In the present article we return to the theme of [5] and establish the following improvement on Theorem 1 above.

THEOREM 2. Let $G$ be a locally soluble-by-finite group in which every subgroup of infinite rank is subnormal. If $G$ has infinite rank then $G$ is soluble, and hence a Baer group.

As noted in [5], the solubility of $G$ is a reasonable hypothesis in the statement of Theorem 1, since a group with all subgroups subnormal is in any case soluble, a remarkable result due to Möhres [6]. On the other hand, such a group need not be nilpotent, as the famous Heineken-Mohamed examples [4] indicate - these groups have trivial centre and all subgroups subnormal. Whether the local hypothesis on $G$ presented in Theorem 2 can be weakened to any significant extent is not clear the conclusion may hold for locally graded groups $G$ i.e. groups in which all finitely generated nontrivial subgroups have nontrivial finite images. However, as it is not even 
known whether a finitely generated $p$-group ( $p$ a prime) that is residually finite, and has all subgroups either finite or of finite index (and hence subnormal), need itself be finite, we are nowhere near being able to answer the above question at present.

Papers [10] and [11] address the topic of groups in which every subgroup is either subnormal or nilpotent, and we shall have occasion here to use the main results of these articles. Indeed, it is shown in [10] that a torsion-free, locally soluble-by-finite group in which every non-nilpotent subgroup is subnormal is itself nilpotent, and since a locally nilpotent group of finite rank that is torsion-free is nilpotent [7, Theorem 6.36], we deduce immediately that a torsion-free locally nilpotent group that satisfies the hypotheses of Theorem 2 is nilpotent. We therefore obtain the following consequence of Theorem 2.

THEOREM 3. Let $G$ be a torsion-free locally soluble-by-finite group in which every subgroup of infinite rank is subnormal. If $G$ has infinite rank then $G$ is nilpotent.

It is also reasonable to ask whether the conclusion of Theorem 2 can be replaced by the stronger one that such a group $G$, if of infinite rank, must have all subgroups subnormal. The next result shows that this is not the case.

THEOREM 4. There exists a metabelian, locally nilpotent group G of infinite rank such that the torsion subgroup $T$ of $G$ has finite rank and contains a non-subnormal subgroup of $G$, but every subgroup that is not contained in $T$ is subnormal in $G$.

Our final result shows that, in one sense, the above example is optimal.

THEOREM 5. Let G be a group that satisfies the hypotheses of Theorem 2, and suppose that the torsion subgroup $T$ of $G$ has infinite rank. Then every subgroup of $G$ is subnormal.

2. The proof of Theorem 2. Let $G$ be a locally soluble-by-finite group in which all subgroups of infinite rank are subnormal, and suppose that $G$ has infinite rank. We wish to show that $G$ is soluble, for then $G$ is a Baer group, by Theorem 1. If $H$ is an arbitrary subgroup of $G$ of infinite rank then $H$ is subnormal in $G$, as is every subgroup that contains $H$, and by considering a (finite) subnormal series from $H$ to $G$ we see that every factor of this series is soluble [6], so that some term of the derived series of $G$ is contained in $H$. Now, since $G$ has infinite rank it has a locally soluble subgroup $H$ of infinite rank [2], and if $H$ is soluble then so is $G$. Thus we may assume that $G$ is locally soluble. If $G$ is not soluble then it has finitely generated subgroups of arbitrarily high derived length and rank, and so we may also suppose that $G$ is countable.

We now proceed to establish some properties that will allow us to pass to successive sections of $G$ as our proof proceeds. If $H$ is an infinite rank subgroup of any section $K$ of $G$ then $K / \operatorname{Core}_{K}(H)$ is soluble, as above. In particular, if $H$ is soluble then so is $K-$ in other words, each insoluble section $K$ of $G$ has no soluble subgroups of infinite rank. Now a locally nilpotent group of infinite rank has an infinite rank abelian subgroup [7, Corollary 2 to Theorem 6.36], and the same holds for hyperabelian groups [1] and locally finite groups [12]. Again let $K$ be an infinite rank section of $G$. If $M$ is an infinite rank subgroup of $K$ generated by $K$-invariant subgroups $M_{\lambda}$ of finite rank then, since each $M_{\lambda}$ has a characteristic and hence $K$-invariant abelian series [7, Lemma 10.39], $M$ is hyperabelian and $K$ is therefore soluble (since $M$ has an abelian subgroup of infinite rank). On the other hand, if $M$ is a normal subgroup of $K$ of finite rank such that $K / M$ is soluble then $K$ is hyperabelian and therefore soluble. What all this means in 
the present context is that, in order to establish that $G$ is soluble, we may restrict our attention to the following case.

(1) G has no nontrivial normal subgroups of finite rank, and both the locally nilpotent and locally finite radicals of $G$ are trivial. $G$ has no infinite rank soluble subgroups.

Note that hypotheses (1) imply that $G$ is insoluble.

LEMMA 1. G is locally of finite rank, and every proper image of $G$ is soluble and locally nilpotent.

Proof. Let $F$ be a finitely generated subgroup of $G$; then $F$ is soluble and therefore, by (1), of finite rank. If $N$ is an arbitrary nontrivial normal subgroup of $G$ then, again by (1), $N$ has infinite rank and so all subgroups of $G / N$ are subnormal. Thus $G / N$ is locally nilpotent and, by [6], soluble.

Next, let $G_{0}$ denote the intersection of all normal subgroups $N$ of $G$ such that $G / N$ is torsion-free and locally nilpotent. Certainly $G / G_{0}$ is torsion-free, and if $G_{0} \neq 1$ then $G / G_{0}$ is soluble, by Lemma 1 . If $M$ is a proper nontrivial normal subgroup of $G_{0}$ then $M$ has infinite rank, for otherwise $M$ has a characteristic abelian series (as before) and hence contains a nontrivial abelian subnormal subgroup of $G$, contradicting the fact that the locally nilpotent radical of $G$ is trivial. But $M$ of infinite rank implies that $G_{0} / M$ is soluble, so there is a $G$-invariant subgroup $L$ of $M$ with $G / L$ soluble and hence locally nilpotent. If $U / L$ denotes the torsion subgroup of $G_{0} / L$ then we have $G / U$ torsion-free and locally nilpotent, which implies that $U=G_{0}$. Thus all proper images of $G_{0}$ are periodic (in the case $G_{0} \neq 1$ ). On the other hand, if $G_{0}=1$ then $G$ is residually torsion-free nilpotent.

LEMMA 2. Let $X$ be a locally soluble group that is residually torsion-free nilpotent. If $X$ is locally of finite rank then $X$ is locally nilpotent.

Proof. Let $F$ be a finitely generated subgroup of $X$; then $F$ too is residually torsionfree nilpotent. Since $F$ has finite Hirsch length there is no infinite descending chain of normal subgroups of $F$ with each factor being nontrivial and torsion-free. It follows that $F$ is nilpotent, as required.

From Lemma 2 we see that if $G_{0}=1$ then $G$ is locally nilpotent, contradicting (1). Thus $G_{0}$ is also a counterexample to Theorem 2, and we may assume that

(2) every proper image of $G$ is periodic, soluble and locally nilpotent.

The intersection of all nontrivial normal subgroups of $G$ cannot be nontrivial, as a minimal normal subgroup of a locally soluble group is abelian. It follows that $G$ is residually periodic. Since $G$ is countable it is the ascending union of finitely generated subgroups $F_{1} \leq F_{2} \leq \ldots$, and each $F_{i}$ is soluble of finite rank, by Lemma 1 , hence minimax, by [7, Theorem 10.38], and therefore nilpotent-by-abelian-by-finite [7, Proof of Theorem 10.38]. So, for each $i$, there are normal subgroups $U_{i}, V_{i}$ of $F_{i}$ with $V_{i} \leq U_{i}$, $V_{i}$ nilpotent, $U_{i} / V_{i}$ abelian and $F_{i} / U_{i}$ finite. $V_{i}$ may be chosen to be the Fitting radical of $F_{i}$ (in this case, the unique maximal normal nilpotent subgroup of $F_{i}$ ), and $U_{i} / V_{i}$ may be assumed torsion-free for each $i$. Since $G$ is residually periodic, each $F_{i}$ is residually finite. Let $V=\left\langle V_{i}: i=1,2,3, \ldots\right\rangle$.

LEMMA 3. If $U_{1} / V_{1}$ is nontrivial then $V$ has finite rank.

Proof. Suppose for a contradiction that $V$ has infinite rank. Then $V$ is subnormal in $G$, as is every subgroup that contains $V$, and so some term $D$ of the derived series 
of $G$ is contained in $V$, while $G / D$ is periodic, by (2). Choose $u \in U_{1} \backslash V_{1}$. Then there exists a positive integer $n$ such that $u^{n} \in V \cap F_{1}$, and so $u^{n} \in\left(V_{1} \ldots V_{r}\right) \cap F_{1}$ for some integer $r$. Now, for all $j>1,\left(V_{1} \ldots V_{j}\right) \cap F_{1}=\left(V_{1} \ldots V_{j}\right) \cap F_{j-1} \cap F_{1}=$ $\left(V_{1} \ldots V_{j-1}\right)\left(V_{j} \cap F_{j-1}\right) \cap F_{1}=\left(V_{1} \ldots V_{j-1}\right) \cap F_{1}$, since $V_{j} \cap F_{j-1}$ is a normal nilpotent subgroup of $F_{j-1}$ and hence contained in $V_{j-1}$. It follows that $\left(V_{1} \ldots V_{r}\right) \cap F_{1}=V_{1}$, and we obtain the contradiction that $U_{1} / V_{1}$ is periodic. The lemma is proved.

Thus, if $U_{1} / V_{1}$ is nontrivial then $V$ has finite rank $r$, say, and so each $V_{i}$ has rank (at most) $r$. We proceed to dispense with this possibility. Firstly, suppose that $G$ is not torsion-free. Then there is an element $x$ of prime order $p$ in $G$, and $\langle x\rangle^{G}$ has infinite rank and is insoluble, by (1). We may assume in this case that $G$ is generated by elements of order $p$, and so every proper image of $G$ is a locally nilpotent $p$-group, by (2). In particular, $G$ is residually a $p$-group and so every finitely generated subgroup is residually finite- $p$. Thus, in general, we may suppose that every periodic subgroup is a $p$-group, where $p$ is some fixed prime.

For each $i$, let $T_{i}$ denote the torsion subgroup of $V_{i}$, and let $S$ be the subgroup of $V$ generated by all $T_{i}$. Clearly $S$ is a locally finite $p$-group and, since it has finite rank, $S$ is Černikov. But $S$ is also residually of finite exponent, so it is finite of order $m$, say, and each $T_{i}$ therefore has order at most $m$. For each $i$, let $D_{i}$ be the centralizer of $T_{i}$ in $F_{i}$, so that $F_{i} / D_{i}$ has bounded order and therefore bounded derived length.

Now $V_{i} / T_{i}$ is a torsion-free nilpotent group of rank at most $r$ and hence of nilpotency class $c$ that is also at most $r$, as may be seen by noting that the rank of $V_{i} / T_{i}$ is the sum of the ranks of the upper central factors of $V_{i} / T_{i}$. Let $\bar{Z}_{i, j}$ denote one such upper central factor, and let $C_{i, j}$ be the centralizer of $\bar{Z}_{i, j}$ in $F_{i}$; then $F_{i} / C_{i, j}$ embeds in $G L(r, \mathbb{Q})$ and therefore has $r$-bounded derived length, by the well-known theorem of Zassenhaus [7, Theorem 3.2.3]. Denoting by $C_{i}$ the intersection of the $C_{i, j}, j=1, \ldots, c$, we deduce that $F_{i} / C_{i}$ has bounded derived length, as therefore has $F_{i} / E_{i}$, where $E_{i}=C_{i} \cap D_{i}$. If $E_{i} V_{i} / V_{i}$ is non-trivial then, since $F_{i} / V_{i}$ is soluble, there is a nontrivial $F_{i}$-invariant abelian subgroup $B_{i} V_{i} / V_{i}$ of $E_{i} V_{i} / V_{i}$. But $B_{i}$ acts nilpotently on $V_{i}$ and so $B_{i} V_{i}$ is nilpotent, and since $V_{i}$ is the Fitting radical of $F_{i}$ we have a contradiction. It follows that $E_{i}$ is contained in $V_{i}$ and hence that $F_{i} / V_{i}$ has bounded derived length, so that $F_{i}$ also has bounded derived length. But this gives the contradiction that $G$ is soluble.

The above argument establishes that $V$ cannot have finite rank, and Lemma 3 now tells us that $U_{1} / V_{1}$ is trivial. But if $U_{i}>V_{i}$ for any $i$ then a suitable relabelling allows us to suppose that $U_{1}>V_{1}$, and we are thus forced to conclude that $U_{i}=V_{i}$ for all $i$, so each $F_{i}$ is finitely generated nilpotent-by-finite and hence polycyclic. We know from (1) that every abelian subgroup of $G$ has finite rank; if the ranks of the torsion-free abelian subgroups of $G$ were bounded then we would obtain from [2, Corollary 3.7] that $G$ has finite rank. By this contradiction we see that there is no bound for the torsion-free ranks of the $F_{i}$.

Lemma 4. Let $X$ be a polycyclic group, $Y$ a subgroup of $X$, and let $V$ be a normal subgroup of finite index in $Y$. Suppose that the derived length of $X$ is exactly $d$. Then there is a normal subgroup $U$ of finite index in $X$ such that $X / U$ has derived length exactly $d$ and $U \cap Y \leq V$.

Proof. Since $X$ is residually finite there is certainly a normal subgroup $U_{0}$ of finite index in $X$ with the derived length of $X / U_{0}$ being exactly $d$. Also, using a theorem of Mal'cev [8, 5.4.16], there is a subgroup $U_{1}$ of finite index in $X$ such that $U_{1} \cap Y=V$. Let $U_{2}$ be the core of $U_{1}$ in $X$ and set $U=U_{0} \cap U_{2}$. 
Conclusion of the proof of Theorem 2. For an arbitrary group $H$, we denote by $H^{(n)}$ the $n$th term of the derived series of $H$, and if $H$ is soluble we denote by $d(H)$ the derived length of $H$. Suppose that $d\left(F_{1}\right)=d_{1}$ and choose $N_{1}$ normal in $F_{1}$ with $F_{1} / N_{1}$ finite and $d\left(F_{1} / N_{1}\right)=d_{1}$. By relabelling if necessary we may assume that $d\left(F_{2}\right)=d_{2}$ is greater that $2 d_{1}$, and by Lemma 4 we may choose $N_{2} \triangleleft F_{2}$ with $F_{2} / N_{2}$ finite, $d\left(F_{2} / N_{2}\right)=d_{2}$ and $N_{2} \cap F_{1} \leq N_{1}$. Since $d\left(N_{1} N_{2} / N_{2}\right) \leq d\left(N_{1}\right) \leq d_{1}$, we see that $F_{2}^{\left(d_{1}\right)} \not \leq N_{1} N_{2}$. Note that $N_{1} N_{2} \cap F_{1}=N_{1}\left(N_{2} \cap F_{1}\right)=N_{1}$. Next, we may assume that $d\left(F_{3}\right)=d_{3}>2 d_{2}+$ $d_{1}$, and so there exists a normal subgroup $N_{3}$ of $F_{3}$ with $F_{3} / N_{3}$ finite, $d\left(F_{3} / N_{3}\right)=$ $d_{3}$ and $N_{3} \cap F_{2} \leq N_{2}$. We have $d\left(N_{1} N_{2} N_{3} / N_{3}\right) \leq d\left(N_{1} N_{2}\right) \leq d_{1}+d_{2}$, so that $F_{3}^{\left(d_{2}\right)} \not \leq$ $N_{1} N_{2} N_{3}$, also $N_{1} N_{2} N_{3} \cap F_{2} \leq N_{1} N_{2}$. We continue in this manner, and with the obvious notation. Write $J_{i}=N_{1} \ldots N_{i}$ for each $i$, and set $J=\left\langle J_{i}: i=1,2, \ldots\right\rangle$. Since the $F_{i}$ have unbounded torsion-free rank, so do the $N_{i}$, and it follows that $J$ has infinite rank and hence that every subgroup containing $J$ is subnormal in $G$. We deduce that $G^{(d)} \leq J$ for some integer $d$, and hence that $F_{r+1}^{(d r)} \leq J$ for some $r$ (chosen so that $d_{r} \geq d$ ). Certainly therefore $F_{r+1}^{\left(d_{r}\right)} \leq J_{i}$ for some $i>r+1$. But $J_{i} \cap F_{r+1}=\left(N_{1} \ldots N_{i}\right) \cap F_{r+1} \leq$ $\left(N_{1} \ldots N_{i}\right) \cap F_{i-1}=\left(N_{1} \ldots N_{i-1}\right)\left(N_{i} \cap F_{i-1}\right)=J_{i-1}$, and continuing (if necessary) we see that $F_{r+1}^{\left(d_{r}\right)}$ is contained in $J_{r+1}$, contradicting the choice of $N_{r+1}$. The theorem is therefore proved.

3. The proof of Theorem 5. Let $G$ be as stated, so that $G$ is both soluble and a Baer group, by Theorem 2. Note that $G / T$ is nilpotent, by the remarks preceding the statement of Theorem 3. Assuming for a contradiction that not every subgroup of $G$ is subnormal, there is a subgroup $H$ of finite rank that is not subnormal in $G$. If every $p$ component of $T$ has finite rank then, since $T$ has infinite rank, we may write $T=T_{1} \times$ $T_{2}$ for some infinite rank subgroups $T_{1}, T_{2}$, where the sets of primes involved in $T_{1}$ and $T_{2}$ are disjoint. Since $G / T$ is nilpotent and each of $H T_{1}, H T_{2}$ is subnormal in $G$, there is a positive integer $n$ such that $\left[G,_{n} H\right] \leq T \cap H T_{1} \cap H T_{2}=T_{1}(T \cap H) \cap T_{2}(T \cap H)=$ $T_{1}\left(T_{2} \cap H\right) \cap T_{2}\left(T_{1} \cap H\right)=\left(T_{2} \cap H\right)\left(T_{1} \cap H\right) \leq H$, giving the contradiction that $H$ is subnormal. Hence there is a $p$-component $P$ of $T$ that has infinite rank, and since $H P$ is subnormal in $G$ we may suppose that $G=H P$. There is a term $R$ of the derived series of $P$ that has infinite rank while $R^{\prime}$ has finite rank, and so there exist $G$-invariant subgroups $A$ and $B$ of $P$, with $B$ of finite rank and contained in $A$ and $A / B$ infinite abelian and of exponent $p$. Since every subgroup of $G / A$ is subnormal, we may further suppose that $G=A H$.

Let $D$ denote the divisible component of $B$; then $D$ is central in the Baer $p$-group $A$ [7, Lemma 3.13] and hence, as $D H$ is a Baer group of finite rank $r$, say, we have $D \leq Z_{r}(G)$, the $r$ th term of the upper central series of $G$ [7, Vol. 2, p. 38]. But $B / D$ is finite, so $B \leq Z_{s}(G)$ for some integer $s$, and it follows that $H$ is subnormal in $H B$, so we may assume that $B$ is contained in $H$. But now $H \cap A$ is normal in $G$, and we may again factor and hence suppose that $A$ is abelian and of exponent $p$. By induction on the derived length of $H$ we may assume that $H^{\prime}$ is subnormal in $G$, so that $\left[A,{ }_{k} H^{\prime}\right] \leq H^{\prime} \cap A=1$, for some positive integer $k$. Writing $A_{i}=\left[A,{ }_{i} H^{\prime}\right]$ for each $i \geq 0$ (where $A_{0}=A$ ), we see that $H A_{i+1}$ is not subnormal in $H A_{i}$ for some integer $i$; certainly $A_{i} / A_{i+1}$ must be infinite, and so factoring once more allows us to suppose that $\left[A, H^{\prime}\right]=1$. This in turn means that $H^{\prime}$ is normal in $G$, so we set $H^{\prime}=1$ and assume that $H$ is abelian.

Since $H$ has finite rank, it has a finitely generated subgroup $F$ such that $H / F$ is periodic. Because $G$ is a Baer group there is a positive integer $m$ such that $\left[A,{ }_{m} F\right]=1$, 
and repeating the above argument with $F$ in place of $H^{\prime}$ we reduce to the case where $H$ is itself periodic. But the $p^{\prime}$-component of $H$ may clearly be assumed trivial, so that now $G$ is a $p$-group. Let $S$ be an arbitrary subgroup of $G$. If $S$ has finite rank then, since it is also a Baer p-group, $S$ is nilpotent [7, Vol. 2, p. 38], and it follows that every subgroup of the $p$-group $G$ is either nilpotent or subnormal. By [11, Theorem 3] we deduce that every subgroup of $G$ is subnormal, our final contradiction.

4. The proof of Theorem 4. Let $p_{1}$ be an arbitrary prime and define inductively a set of primes $p_{1}, p_{2}, \ldots$ such that $p_{n+1}>n\left(p_{1}+\ldots+p_{n}\right)$ for each $n \geq 1$. For each $n$, let $H_{n}=\left\langle c_{n}\right\rangle \rtimes\left\langle b_{n}\right\rangle$, where $\left|c_{n}\right|=p_{n}^{n+1},\left|b_{n}\right|=p_{n}^{n}$ and $\left[c_{n}, b_{n}\right]=c_{n}^{p_{n}}$, so that $H_{n}$ is a finite $p$-group of nilpotency class $n$. Let $K$ denote the cartesian product of the $H_{n}$, and let $T$ be the torsion subgroup of $K$, namely their direct product. Write $d_{1}=$ $\left(1, c_{2}^{p_{1}}, c_{3}^{p_{2}}, c_{4}^{p_{3}}, \ldots\right), d_{2}=\left(1,1, c_{3}^{p_{1}}, c_{4}^{p_{2}}, \ldots\right), \ldots$ (so that $d_{n}$ has 1 for each of its first $n$ entries, followed by $\left.c_{n+1}^{p_{1}}, c_{n+2}^{p_{2}}, \ldots\right)$. The direct product $D$ of the $\left\langle d_{n}\right\rangle$ is a torsion-free abelian subgroup of $K$ that has infinite rank. Our group $G$ is then the subgroup $T \rtimes D$ of $K$. Setting $C=\operatorname{Dr}\left\langle c_{n}\right\rangle, B=\operatorname{Dr}\left\langle b_{n}\right\rangle$, we have $T=C \rtimes B, G^{\prime}=T^{\prime}[T, D] \leq C$, and so $G$ is metabelian. Clearly $[G, D, D]=1$, and so $D$ is subnormal in $G$, and $G$ is a Baer group. However, $B$ is not subnormal in $G$, since $\left[\left\langle c_{n}\right\rangle_{n-1}\left\langle b_{n}\right\rangle\right] \neq 1$, for each $n$. Finally, $T$ is (non-nilpotent) of rank 2 , and $G$ has infinite rank.

Let $U$ be a subgroup of $G$ not contained in $T$, and choose an element $u$ of $U \backslash T$. Then $u=d_{1}^{\alpha_{1}} d_{2}^{\alpha_{2}} \ldots d_{k}^{\alpha_{k}} t$, for some $t \in T$, some positive integer $k$ and some $\alpha_{1}, \alpha_{2}, \ldots, \alpha_{k} \in \mathbb{Z}$, where not every $\alpha_{i}$ is zero. Certainly $t \in D r_{i=1}^{l} H_{i}$ for some $l$. Choose an integer $N>\max \left(\left|\alpha_{1}\right|+k, \ldots,\left|\alpha_{k}\right|+k, l\right)$, and let $n \geq N$. We have $[G, U] \leq C$, and so it suffices to prove that $U$ is subnormal in $U C$. If $V=D r_{i=1}^{l} C_{i}$ then we see that $V \leq Z_{N+1}(G)$, and so $U$ is subnormal in $U V$. We claim that now $U V$ is normal in $U C$, and to show this we may as well suppose that $V$ is contained in $U$. It remains to show that $\left[\left\langle c_{n}\right\rangle, U\right] \leq U$ for all $n \geq N$.

Let $n$ be an arbitrary integer greater than or equal to $N$. Certainly $D C$ is normal in $G$ (since $G^{\prime} \leq C$ ) and $D C$ centralizes $\left\langle c_{n}\right\rangle$. If $U$ projects trivially onto $\left\langle b_{n}\right\rangle$ via the restriction of the natural epimorphism from $G$ onto $G / D C$ then we have $\left[\left\langle c_{n}\right\rangle, U\right]=1$, and there is nothing to prove. Otherwise, there is an element $v$ of $U$ of the form $v=b_{n}^{p_{n}^{\sigma_{n}}} w$, where $w$ is contained in the ( $G$-invariant) subgroup generated by $D C$ and $D r_{i \neq n}\left\langle b_{i}\right\rangle$, and where $\sigma(n)$, the least such non-negative integer, is less than $n$. Observe that $\left[\left\langle c_{n}\right\rangle, U\right]$ is precisely $\left[\left\langle c_{n}\right\rangle,\left\langle b_{n}^{p_{n}^{\sigma_{n}}}\right\rangle\right]$. It therefore suffices to prove that $g_{n}:=\left[c_{n}, b_{n}^{p_{n}^{\sigma_{n}}}\right]$ is contained in $U$. (Note that this does indeed suffice, since every subgroup of $C$ is normal in $G$, while $g_{n}$ generates $\left[\left\langle c_{n}\right\rangle, U\right]$ as a $G$-group.) Now $U$ contains $[u, v]=\left[d_{1}^{\alpha_{1}} d_{2}^{\alpha_{2}} \ldots d_{k}^{\alpha_{k}} t, b_{n}^{\sigma_{n}^{\sigma_{n}}} w\right]$, and since $G^{\prime} \leq C$, which is the direct product of its primary components, it follows without difficulty that $U$ contains $\left[d_{1}^{\alpha_{1}} d_{2}^{\alpha_{2}} \ldots d_{k}^{\alpha_{k}}, b_{n}^{p_{n}^{\sigma_{n}}}\right]$ (note that $[t, G]$ and $[D, w]$ both intersect $H_{n}$ trivially). So $U$ contains $\left[c_{n}^{\lambda(n)}, b_{n}^{p_{n}^{\sigma_{n}}}\right]$, where $\lambda(n)=\alpha_{1} p_{n-1}+\alpha_{2} p_{n-2}+\ldots+\alpha_{k} p_{n-k}$. It now suffices to prove that $0<|\lambda(n)|<p_{n}$. We have $|\lambda(n)| \leq\left(\max \left(\left|\alpha_{i}\right|\right)\left(p_{n-1}+p_{n-2}+\ldots+p_{n-k}\right)<n\left(p_{n-1}+p_{n-2}+\ldots+p_{n-k}\right)\right.$ $\leq n\left(p_{n-1}+p_{n-2}+\ldots+p_{1}\right)<p_{n}$. Also, if $i$ is least such that $\alpha_{i} \neq 0$, then $\lambda(n)=$ $\alpha_{i} p_{n-i}+\alpha_{i+1} p_{n-i-1}+\ldots+\alpha_{k} p_{n-k}$, and $\left|\alpha_{i} p_{n-i}\right| \geq p_{n-i}>(n-i)\left(p_{n-i-1}+\ldots+p_{1}\right) \geq$ $(n-i)\left(p_{n-i-1}+\ldots+p_{n-k}\right) \geq(n-k)\left(p_{n-i-1}+\ldots+p_{n-k}\right)>\left(\max \left|\alpha_{j}\right|\right)\left(p_{n-i-1}+\ldots+\right.$ $\left.p_{n-k}\right) \geq\left|\alpha_{i+1} p_{n-i-1}+\ldots+\alpha_{k} p_{n-k}\right|$, so $\lambda(n) \neq 0$. Hence $0<|\lambda(n)|<p_{n}$, and the result follows. 


\section{REFERENCES}

1. R. Baer and H. Heineken, Radical groups with finite abelian subgroup rank, Illinois $J$. Math. 16 (1972), 533-580.

2. M. R. Dixon, M. J. Evans and H. Smith, Locally (soluble-by-finite) groups of finite rank, J. Algebra 182 (1996), 756-769.

3. M. J. Evans and Y. Kim, On groups in which every subgroup of infinite rank is subnormal of bounded defect, preprint.

4. H. Heineken and I. J. Mohamed, A group with trivial centre satisfying the normalizer condition, J. Algebra 10 (1968), 368-376.

5. L. A. Kurdachenko and P. Soules, Groups with all non-subnormal subgroups of finite rank, in Groups, St. Andrews, Proceedings (2001), to appear.

6. W. Möhres, Auflösbarkeit von Gruppen, deren Untergruppen alle subnormal sind, Arch. Math. (Basel) 54 (1990), 232-235.

7. D. J. S. Robinson, Finiteness conditions and generalized soluble groups (2 vols.) (SpringerVerlag, 1972).

8. D. J. S. Robinson, A course in the theory of groups (Springer-Verlag, 1982).

9. J. E. Roseblade, On groups in which every subgroup is subnormal, J. Algebra 2 (1965), $402-412$.

10. H. Smith, Torsion-free groups with all non-nilpotent subgroups subnormal, in Topics in Infinite Groups, Quaderni di Matematica 8 (2001), 297-308.

11. H. Smith, Groups with all non-nilpotent subgroups subnormal, in Topics in Infinite Groups, Quaderni di Matematica 8 (2001), 309-326.

12. V. P. Sunkov, On locally finite groups of finite rank, Algebra and Logic 10 (1971), $127-142$. 